\title{
CONFORMAL LOWER BOUNDS FOR THE DIRAC OPERATOR OF EMBEDDED HYPERSURFACES*
}

\author{
OUSSAMA HIJAZI ${ }^{\dagger}$, SEBASTIÁN MONTIEL ${ }^{\ddagger}$, AND XIAO ZHANG§
}

\begin{abstract}
We find sharp lower bounds for the first nonnegative eigenvalue of the classical intrinsic Dirac operator of a compact hypersurface bounding a domain in a Riemannian spin manifold. These estimates are given in terms of scalar (spectral) conformal invariants of the enclosed domain which are involved in the solution of the Yamabe problem.
\end{abstract}

1. Introduction. In [Hij1], the first author used the conformal behavior of the nonzero eigenvalues $\lambda$ of the Dirac operator to show that on a closed connected spin manifold $\lambda^{2}$ is, up to a dimensional constant, at least equal to the first eigenvalue of the Yamabe operator.

Using techniques for pseudo-differential operators and Sobolev embeddings, J. Lott [Lo] proved the existence of conformal lower bounds for $\lambda^{2}$. In [Hij2] it is shown that the Yamabe number, i.e., the infimum over a conformal class of metrics of the normalized total scalar curvatures, gives such a lower bound.

In [HMZ1], the present authors considered a domain $\Omega$ with boundary $\Sigma$ inside a compact $(n+1)$-dimensional spin manifold $M$ and showed that if $M$ has nonnegative scalar curvature, then the first nonnegative eigenvalue $\lambda_{1}$ of the intrinsic Dirac operator of $\Sigma$ satisfies

$$
\lambda_{1} \geq \frac{n}{2} \inf _{\Sigma} H
$$

where $H$ is the mean curvature. The main new ingredient was to use the boundary condition of Atiyah-Patodi-Singer (APS) type for Dirac operators. If the ambient space has nonnegative Einstein tensor, (1.1) improves Friedrich's inequality for embedded hypersurfaces. As an application, a spinorial proof of the classical Alexandrov Theorem was obtained.

The present paper is devoted to the conformal aspect of the results obtained in [HMZ1]. We improve (1.1) by showing that

$$
\lambda_{1} \geq \frac{n}{2} \nu_{1}(B)
$$

where $\nu_{1}(B)$ is the first eigenvalue of the conformal mean curvature operator $B$ (see Theorem 9 for a precise statement). Here we don't need to assume that $M$ has nonnegative scalar curvature. Furthermore, the limitting-case of (1.2) is characterized by the existence of a parallel spinor on the ambient manifold for a metric in the conformal class. We then use the Hölder inequality to show that

$$
\lambda_{1} \geq \frac{n}{2} \frac{\mathcal{Q}(\Omega, \Sigma)}{\operatorname{vol}(\Sigma)^{\frac{1}{n}}}
$$

\footnotetext{
${ }^{*}$ Received May 23, 2001; accepted for publication June 14, 2001. This work is partially done during the second and third authors visit to the Institut Élie Cartan, Université Henri Poincaré, Nancy 1, and they would like to thank the institute for its hospitality.

†Institut Élie Cartan, Université Henri Poincaré, Nancy I, B.P. 239, 54506 Vandœuvre-Lès-Nancy Cedex, France (hijazi@iecn.u-nancy.fr).

‡Departamento de Geometría y Topología, Universidad de Granada, 18071 Granada, Spain (smontiel@goliat.ugr.es). The author is partially supported by a Spanish MCyT grant No. BFM2001-2967.

$\S$ Institute of Mathematics, Academy of Mathematics and Systems Sciences, Chinese Academy of Sciences, Beijing 100080, P.R. China (xzhang@math.ac.cn). Research of X.Z. is partially supported by the Chinese NSF and mathematical physics program of CAS.
} 
where $\mathcal{Q}(\Omega, \Sigma)$ is the boundary Yamabe conformal invariant (see Corollary 11).

For Dirac operators, the APS boundary condition is not conformally invariant, while the classical local boundary conditions are indeed conformally invariant but do not exist in all dimensions and there are topological obstructions for their existence [BW, GLP, HMZ2, Se]. In this paper, we find a new local conformal boundary condition which exists in all dimensions (see Section 5). The key point to establish (1.2) is to solve a boundary problem for Dirac operators with such boundary condition.

We point out that if the scalar curvature of the domain is nonnegative, then $\nu_{1}(B) \geq \inf _{\Sigma} H$ and for the Clifford torus embedded in $\mathbb{R}^{3}, \nu_{1}(B)>0$ while $\inf _{\Sigma} H<$ 0 (see Remark 4).

Finally, we would like to mention a series of relevant results by Escobar concerning the lower bound estimates for the first non-zero Steklov eigenvalue of the Laplacian operator [Es2, Es3, Es4], and further estimates for the Steklov problem on minimizing metrics for the Sobolev trace quotient by Araujo [Ar].

2. Preliminaries on spin manifolds. Let $(M,\langle\rangle$,$) be an (n+1)$-dimensional Riemannian spin manifold and denote by $\bar{\nabla}$ the Levi-Civita connection on the tangent bundle $T M$. We fix a spin structure on $M$ and denote by $\operatorname{Spin}(M)$ the corresponding principal bundle with structural group the spinor group $\operatorname{Spin}(n+1)$. The spinor bundle $\mathbb{S} M=\operatorname{Spin}(M) \times{ }_{\gamma_{n+1}} \mathbb{S}_{n+1}$ on $M$ is the associated complex $2^{\left[\frac{n+1}{2}\right]}$ dimensional complex vector bundle. This representation provides a left Clifford multiplication

$$
\gamma: \mathbb{C} \ell(M) \longrightarrow \operatorname{End}(\mathbb{S} M)
$$

which is a fibre preserving algebra morphism. Then $\mathbb{S} M$ becomes a bundle of complex left modules over the Clifford bundle $\mathbb{C} \ell(M)$ over the manifold $M$. When $n+1$ is even, the spinor bundle has the decomposition

$$
\mathbb{S} M=\mathbb{S} M^{+} \oplus \mathbb{S} M^{-} .
$$

where $\mathbb{S} M^{ \pm}$are the \pm 1 -eigenspaces of the endomorphism $\gamma_{n+1}\left(\omega_{n+1}\right)$, with $\omega_{n+1}=$ $i^{\left[\frac{n+2}{2}\right]} e_{1} \cdot e_{2} \cdots e_{n+1}$ the complex volume form.

On the spinor bundle $\mathbb{S} M$, one has (see [LM]) a natural Hermitian metric, denoted as the Riemannian metric by $\langle$,$\rangle , and the Spinorial Levi-Civita connection \bar{\nabla}$ acting on spinor fields. The Hermitian metric and $\bar{\nabla}$ are compatible with the Clifford multiplication (2.1). That is

$$
\begin{gathered}
X\langle\psi, \varphi\rangle=\left\langle\bar{\nabla}_{X} \psi, \varphi\right\rangle+\left\langle\psi, \bar{\nabla}_{X} \varphi\right\rangle \\
\langle\gamma(X) \psi, \gamma(X) \varphi\rangle=|X|^{2}\langle\psi, \varphi\rangle \\
\bar{\nabla}_{X}(\gamma(Y) \psi)=\gamma\left(\bar{\nabla}_{X} Y\right) \psi+\gamma(Y) \bar{\nabla}_{X} \psi
\end{gathered}
$$

for any spinor fields $\psi, \varphi \in \Gamma(\mathbb{S} M)$ and any tangent vector fields $X, Y \in \Gamma(T M)$. Since $\bar{\nabla} \omega_{n+1}=0$, so when $n+1$ is even, the decomposition (2.2) becomes orthogonal and $\bar{\nabla}$ preserves this decomposition.

The Dirac operator $\bar{D}$ on $\mathbb{S} M$ is the first order elliptic differential operator locally given by

$$
\bar{D}=\sum_{i=1}^{n+1} \gamma\left(e_{i}\right) \bar{\nabla}_{e_{i}}
$$


where $\left\{e_{1}, \ldots, e_{n+1}\right\}$ is a local orthonormal frame of $T M$. When $n+1$ is even, the Dirac operator interchanges positive and negative spinor fields, that is,

$$
\bar{D}: \Gamma\left(\mathbb{S} M^{ \pm}\right) \longmapsto \Gamma\left(\mathbb{S} M^{\mp}\right)
$$

3. Hypersurfaces and induced structures. In this section, we compare the restriction of the spinor bundle of a spin manifold $M$ to an orientable hypersurface $\Sigma \subset$ $M$ and its Dirac-type operator to the intrinsic spinor bundle of $\Sigma$ and its fundamental Dirac operator. These facts are well-known (see for example [Bu, Tr, Bä1, BFGK]). For completeness, we introduce the key facts.

We have an induced Riemannian metric on $\Sigma$ and let $\nabla$ be its Levi-Civita connection. The Gauss formula says that

$$
\nabla_{X} Y=\bar{\nabla}_{X} Y-\langle A X, Y\rangle N
$$

where $X, Y$ are vector fields tangent to the hypersurface $\Sigma$, the vector field $N$ is a global unit field normal to $\Sigma$ and $A$ stands for the shape operator corresponding to $N$, that is,

$$
\bar{\nabla}_{X} N=-A X, \quad \forall X \in \Gamma(T \Sigma) \text {. }
$$

Recall that the spin structure of $M$ induces on $\Sigma$ in the following way. By the map $\left(e_{1}, \ldots, e_{n}\right) \longmapsto\left(e_{1}, \ldots, e_{n}, N\right)$ it is possible to identify the principal $\mathrm{SO}(n)$-bundle of oriented orthonormal frames on the hypersurface $\Sigma$ with a subbundle of the restriction to $\Sigma$ of the bundle of oriented orthonormal frames on $M$. Pulling back the bundle $\operatorname{Spin}(M)_{\mid \Sigma}$ via this map, one obtains a spin structure $\operatorname{Spin}(\Sigma)$ on $\Sigma$. In fact the group $\operatorname{Spin}(n) \subset \mathbb{C} \ell_{n}^{0}$ acts on the restricted bundle $\operatorname{Spin}(M)_{\mid \Sigma}$ via the identification

$$
\begin{aligned}
\alpha: \mathbb{C} \ell_{n}=\mathbb{C} \ell_{n}^{0} \oplus \mathbb{C} \ell_{n}^{1} & \longrightarrow \mathbb{C} \ell_{n+1}^{0} \subset \mathbb{C} \ell_{n+1} \\
\eta^{0}+\eta^{1} & \longmapsto \eta^{0}+\eta^{1} \cdot N
\end{aligned}
$$

between the $n$-dimensional Clifford algebra and the even part $\mathbb{C} \ell_{n+1}^{0}$. Hence we have that the restriction

$$
\mathbf{S} \Sigma:=\mathbb{S} M_{\mid \Sigma}=\operatorname{Spin}(\Sigma) \times_{\gamma_{n+1} \circ \alpha} \mathbb{S}_{n+1}
$$

is a left module over $\mathbb{C} \ell(\Sigma)$ with Clifford multiplication

$$
\gamma_{\Sigma}: \mathbb{C} \ell(\Sigma) \longrightarrow \operatorname{End}(\mathbf{S} \Sigma)
$$

given by $\gamma_{\Sigma}=\gamma \circ \alpha$. That is,

$$
\gamma_{\Sigma}(X) \psi=\gamma(X) \gamma(N) \psi
$$

for every $\psi \in \Gamma(\mathbf{S} \Sigma)$ and $X \in \Gamma(T \Sigma)$. Consider on $\mathbf{S} \Sigma$ the Hermitian metric $\langle$, induced from that of $\mathbb{S} M$. This metric immediately satisfies the compatibility condition (2.4) if one puts on $\Sigma$ the Riemannian metric induced from $M$ and the Clifford multiplication $\gamma_{\Sigma}$ defined in (3.4). Now the Gauss formula (3.1) implies that the spin connection $\nabla$ on $\mathbf{S} \Sigma$ is given by the following spinorial Gauss formula

$$
\nabla_{X} \psi=\bar{\nabla}_{X} \psi-\frac{1}{2} \gamma_{\Sigma}(A X) \psi=\bar{\nabla}_{X} \psi-\frac{1}{2} \gamma(A X) \gamma(N) \psi
$$


for every $\psi \in \Gamma(\mathbf{S} \Sigma)$ and $X \in \Gamma(T \Sigma)$. Note that the compability conditions (2.3), (2.4) and (2.5) are satisfied for $\left(\mathbf{S} \Sigma, \gamma_{\Sigma},\langle\rangle,, \nabla\right)$.

Denote by $\mathbf{D}: \Gamma(\mathbf{S} \Sigma) \rightarrow \Gamma(\mathbf{S} \Sigma)$ the Dirac operator associated with the Dirac bundle $\mathbf{S} \Sigma$ over the hypersurface. It is a well known fact that $\mathbf{D}$ is a first order elliptic differential operator which is formally $L^{2}$-selfadjoint. By (3.5), for any spinor field $\psi \in \Gamma(\mathbf{S} \Sigma)$, we have

$$
\mathbf{D} \psi=\sum_{j=1}^{n} \gamma_{\Sigma}\left(e_{j}\right) \nabla_{e_{j}} \psi=\frac{n}{2} H \psi-\gamma(N) \sum_{j=1}^{n} \gamma\left(e_{j}\right) \bar{\nabla}_{e_{j}} \psi,
$$

where $\left\{e_{1}, \ldots, e_{n}\right\}$ is a local orthonormal frame of $T \Sigma$ and $H=\frac{1}{n} \operatorname{trace} A$ is the mean curvature of $\Sigma$ corresponding to the orientation $N$. From (3.6), if $\psi \in \Gamma(\mathbb{S} M)$ is a spinor field on the ambient manifold $M$, it follows

$$
\mathbf{D} \psi=\frac{n}{2} H \psi-\gamma(N) \bar{D} \psi-\bar{\nabla}_{N} \psi
$$

(note that a spinor field on the ambient manifold $M$ and its restriction to the hypersurface $\Sigma$ are denoted by the same symbol).

LEMMA 1. For any spinor field $\psi \in \Gamma(\mathbf{S} \Sigma)$ and any tangent vector field $X \in$ $\Gamma(T \Sigma)$, the following relations hold

$$
\begin{aligned}
\nabla_{X}(\gamma(N) \psi) & =\gamma(N) \nabla_{X} \psi \\
\mathbf{D}(\gamma(N) \psi) & =-\gamma(N) \mathbf{D} \psi
\end{aligned}
$$

The proof is straightforward using (3.5) and (3.2). As we have mentioned, the aim in this section is to relate the induced Dirac bundle $\mathbf{S} \Sigma$ over the hypersurface and its Dirac operator $\mathbf{D}$ to the intrinsic spinor bundle

$$
\mathbb{S} \Sigma=\operatorname{Spin}(\Sigma) \times_{\gamma_{n}} \mathbb{S}_{n}
$$

over $\Sigma$ and its Dirac operator $D$. For this purpose, we gather in the following proposition, well-known results that we will need later.

Proposition 2. Let $M$ be an $(n+1)$-dimensional Riemannian spin manifold and $(\mathbb{S} M, \gamma)$ its (complex) spinor bundle, where $\gamma: \mathbb{C} \ell(M) \rightarrow \operatorname{End}(\mathbb{S} M)$ denotes the corresponding Clifford multiplication. Consider an orientable hypersurface $\Sigma$ of $M$ and let $\left(\mathbf{S} \Sigma, \gamma_{\Sigma}\right)$ and $(\mathbb{S} \Sigma, \gamma)$ be respectively the induced Dirac bundle and the spinor bundle of the induced spin structure on $\Sigma$. Denote by $\mathbf{D}$ and $D$ the corresponding Dirac operators.

a) When the dimension $n$ of $\Sigma$ is even we have $\left(\mathbf{S} \Sigma, \gamma_{\Sigma}, \mathbf{D}\right) \equiv(\mathbb{S} \Sigma, \gamma, D)$ and the decomposition $\mathbf{S} \Sigma=\mathbf{S} \Sigma^{+} \oplus \mathbf{S} \Sigma^{-}$, given by $\mathbf{S} \Sigma^{ \pm}:=\{\eta \in \mathbf{S} \Sigma: i \gamma(N) \eta= \pm \eta\}$, corresponds, up to the above identification, to the chirality decomposition of the spinor bundle $\mathbb{S} \Sigma$. Hence $\mathbf{D}$ interchanges $\mathbf{S} \Sigma^{+}$and $\mathbf{S} \Sigma^{-}$.

b) When $n$ is odd, the decomposition of $\mathbb{S} M$ into positive and negative spinors induces an orthogonal and $\gamma_{\Sigma}, \mathbf{D}$-invariant decomposition $\mathbf{S} \Sigma=\mathbf{S} \Sigma_{+} \oplus \mathbf{S} \Sigma_{-}$, with $\mathbf{S} \Sigma_{ \pm}:=\mathbb{S} M_{\mid \Sigma}^{ \pm}$, in such a way that $\left(\mathbf{S} \Sigma_{ \pm}, \gamma_{\Sigma}, \mathbf{D}_{\mid \mathbf{S} \Sigma_{ \pm}}\right) \equiv(\mathbb{S} \Sigma, \pm \gamma, \pm D)$. Moreover, we have the following isomorphisms: $\gamma(N): \mathbf{S} \Sigma_{ \pm} \longmapsto \mathbf{S} \Sigma_{\mp}$.

Furthermore, if $\Sigma$ is compact without boundary, then

c) Spec $\mathbf{D}$ is symmetric with respect to zero. 
d) If $\operatorname{dim} \Sigma$ is even, we have $\forall \lambda \in \operatorname{Spec} D$ : $\operatorname{Spec} \mathbf{D}=\operatorname{Spec} D$, and $\operatorname{mult}_{\mathbf{D}}(\lambda)=$ $\operatorname{mult}_{D}(\lambda)$.

e) If $\operatorname{dim} \Sigma$ is odd, we have $\operatorname{Spec} \mathbf{D}=\operatorname{Spec} D \cup(-\operatorname{Spec} D)$. Moreover,

$$
\begin{gathered}
\lambda \in \operatorname{Spec} D \text { and }-\lambda \notin \operatorname{Spec} D \Longrightarrow \operatorname{mult}_{\mathbf{D}}(\lambda)=\operatorname{mult}_{D}(\lambda), \\
\quad \pm \lambda \in \operatorname{Spec} D \Longrightarrow \operatorname{mult}_{\mathbf{D}}(\lambda)=\operatorname{mult}_{D}(\lambda)+\operatorname{mult}_{D}(-\lambda) .
\end{gathered}
$$

4. Conformal covariance. Consider a positive function $h$ on the Riemannian spin manifold $M$ and the corresponding conformal metric $\langle,\rangle^{\star}=h^{2}\langle$,$\rangle . This yields$ to a bundle isometry between the associated spinor bundles $\mathbb{S} M$ and $\mathbb{S}^{\star} M$. For this reason, the two spinor bundles will be denoted by the same symbol $\mathbb{S} M$. On the other hand, for the corresponding Clifford multiplications and spin connections, one has:

$$
\gamma^{\star}=h \gamma, \quad \bar{\nabla}^{\star}-\bar{\nabla}=-\frac{1}{2 h} \gamma(\cdot) \gamma(\bar{\nabla} h)-\frac{1}{2 h}\langle\cdot, \bar{\nabla} h\rangle .
$$

Now the conformal change of the metric on $M$ produces another conformal change of the induced metric on the hypersurface $\Sigma$ corresponding to the same conformal factor $h^{2}$. We then obtain the following identities relating the Clifford multiplications and the covariant derivatives of $\mathbf{S} \Sigma$ corresponding to the two conformal metrics on the hypersurface:

$$
\begin{aligned}
\gamma_{\Sigma}^{\star} & =h \gamma_{\Sigma}, \quad \gamma^{\star}\left(N^{\star}\right)=\gamma(N) \\
\nabla^{\star}-\nabla & =-\frac{1}{2 h} \gamma_{\Sigma}(\cdot) \gamma_{\Sigma}(\nabla h)-\frac{1}{2 h}\langle\cdot, \nabla h\rangle,
\end{aligned}
$$

where $N^{\star}=(1 / h) N$ is a unit vector field normal to $\Sigma$ with respect to $\langle,\rangle^{\star}$. If we use the symbols $\mathbf{D}$ and $\mathbf{D}^{\star}$ to denote the Dirac operators on $\mathbf{S} \Sigma$ relative to the two conformal metrics on $\Sigma$, we can easily show from (4.2), that for any spinor field $\psi \in \Gamma(\mathbf{S} \Sigma)$, the following identity:

$$
\mathbf{D}^{\star}\left(h^{-\frac{n-1}{2}} \psi\right)=h^{-\frac{n+1}{2}} \mathbf{D} \psi .
$$

This property is analogous to the conformal covariance of the classical Dirac operator of a spinor bundle discovered by Hitchin (see [Hit]). Now it is easy to check [Es1] that

$$
\bar{R}^{\star}=f^{-\frac{n+3}{n-1}} L f, \quad H^{\star}=f^{-\frac{n+1}{n-1}} B f,
$$

where for $n \geq 2$, the conformal factor $h$ has been taken as

$$
h=f^{\frac{2}{n-1}},
$$

for a positive function $f$ defined on $\Omega$. Here $L$ is the Conformal Laplacian and $B$ the Conformal mean curvature operator given by

$$
L u=-\frac{4 n}{n-1} \bar{\Delta} u+\bar{R} u, \quad B u=-\frac{2}{n-1}\langle\bar{\nabla} u, N\rangle+H u,
$$

where $\bar{\nabla}$ and $\bar{\Delta}$ are respectively the gradient and Laplace operators of the original metric on $\Omega$ and $N$ is the inward unit normal field along $\Sigma$ corresponding to the original metric. 

has

In [HMZ1], we proved a spinorial Reilly type inequality: For all $\psi \in \Gamma(\mathbb{S} \Omega)$, one

$$
\begin{gathered}
\int_{\Sigma}\left(\langle\mathbf{D} \psi, \psi\rangle-\frac{n H}{2}|\psi|^{2}\right) d \Sigma \geq \\
\frac{1}{4} \int_{\Omega} \bar{R}|\psi|^{2} d \Omega-\frac{n}{n+1} \int_{\Omega}|\bar{D} \psi|^{2} d \Omega
\end{gathered}
$$

and equality occurs if and only if $\psi$ is a twistor-spinor. Now given a spinor field $\psi \in \Gamma(\mathbb{S} \Omega)$ on the domain $\Omega$, we put

$$
\psi^{\star}=f^{-1} \psi .
$$

From this definition, (4.3) and (4.5) it follows

$$
\left\langle\mathbf{D}^{\star} \psi^{\star}, \psi^{\star}\right\rangle=f^{-\frac{2 n}{n-1}}\langle\mathbf{D} \psi, \psi\rangle
$$

which with inequality (4.7) written w.r.t the metric $\langle,\rangle^{\star}$, after using (4.4), (4.6) and the facts

$$
d \Omega^{\star}=f^{\frac{2 n+2}{n-1}} d \Omega, \quad d \Sigma^{\star}=f^{\frac{2 n}{n-1}} d \Sigma,
$$

we deduce the following conformally spinorial Reilly type inequality

$$
\begin{gathered}
\int_{\Sigma}\left(\langle\mathbf{D} \psi, \psi\rangle-\frac{n}{2}|\psi|^{2} f^{-1} B f\right) d \Sigma \geq \\
\frac{1}{4} \int_{\Omega}|\psi|^{2} f^{-1} L f d \Omega-\frac{n}{n+1} \int_{\Omega}\left|\bar{D}^{\star} \psi^{\star}\right|^{2} d \Omega^{\star},
\end{gathered}
$$

valid for any spinor field $\psi \in \Gamma(\mathbb{S} \Omega)$ and any positive function $f \in C^{\infty}(\Omega)$. Moreover, the equality holds if and only if the spinor field $\psi^{\star}=f^{-1} \psi$ is a twistor-spinor with respect to the conformal metric $\langle,\rangle^{\star}=f^{\frac{4}{n-1}}\langle$,$\rangle .$

5. A local elliptic boundary condition for the Dirac operator. As before, $\Sigma$ is a hypersurface of an $(n+1)$-dimensional Riemannian spin manifold $M$ bounding a compact domain $\Omega$. We define two pointwise projections

$$
P_{ \pm}: \mathbf{S} \Sigma \longrightarrow \mathbf{S} \Sigma
$$

on the induced Dirac bundle over the hypersurface, as follows

$$
P_{ \pm}=\frac{1}{2}\left(\operatorname{Id}_{\mathbf{S} \Sigma} \pm i \gamma(N)\right) \text {. }
$$

Note that, from Proposition 2, when $n$ is even, these are nothing but the projections on the \pm -chirality subbundles $\mathbf{S} \Sigma^{ \pm}$. It is immediate to see that $P_{+}$and $P_{-}$are selfadjoint and orthogonal to each other on every $\mathbf{S} \Sigma_{p}$, with $p \in \Sigma$. Moreover, as a consequence of (4.2), $P_{ \pm}$are conformally invariant. We now show that these operators provide good boundary conditions to solve equations for the Dirac operator $\bar{D}$ of $M$.

Proposition 3. Let $\Omega$ be a compact Riemannian spin manifold with boundary a hypersurface $\partial \Omega=\Sigma$. Then the zero order differential operators $P_{ \pm}$acting on $\mathbf{S} \Sigma$, defined in (5.1) are (local) elliptic boundary conditions, that is, they satisfy the condition of Lopatinsky-Shapiro, for the Dirac operator $\bar{D}$ of $\Omega$. 
Proof. The fact that these operators are (local) elliptic boundary conditions ([BW], Chapter 18) or, in other words, they satisfy the so called condition of Lopatinsky-Shapiro ([Hö]), can be checked by local calculations with the principal symbols of the Dirac operator $\bar{D}$ and the boundary operators $P_{ \pm}$. The symbol $\sigma(\bar{D})$ of $\bar{D}$ is given by

$$
\sigma_{v}(\bar{D}) \eta=i \gamma(v) \eta, \quad v \in T_{p} \Omega, \quad p \in \Omega, \quad \eta \in \mathbb{S} \Omega_{p}
$$

Then, if the point $p$ is taken to be on the boundary $\partial \Omega=\Sigma$, we have

$$
\sigma_{u+s N}(\bar{D}) \eta=i(\gamma(u)+s \gamma(N)) \eta, \quad u \in T_{p} \Sigma, \quad s \in \mathbb{R} .
$$

Fix the point $p \in \Sigma$ and the vector $u \in T_{p} \Sigma$ and replace the parameter $s$ by the partial derivative $-i \partial / \partial t$. We have to look for solutions

$$
w:\left[0,+\infty\left[\longrightarrow \mathbb{S} \Omega_{p}=\mathbf{S} \Sigma_{p}\right.\right.
$$

of the corresponding first order equation

$$
\left(\gamma(u)-i \gamma(N) \frac{\partial}{\partial t}\right) w(t)=0
$$

with asymptotic behaviour

$$
\lim _{t \rightarrow+\infty} w(t)=0 .
$$

One can easily see that those solutions are of the form

$$
w(t)=e^{i t \gamma(N) \gamma(u)} \eta, \quad \eta \in \mathbf{S} \Sigma_{p}
$$

where $w(0)=\eta$ has to be an eigenvector of $i \gamma(N) \gamma(u)$ corresponding to a negative eigenvalue, that is,

$$
i \gamma(N) \gamma(u) \eta=-|u| \eta
$$

The ellipticity condition of Lopatinsky-Shapiro requires that the symbol

$$
\sigma_{u}\left(P_{ \pm}\right)=P_{ \pm}: \mathbf{S} \Sigma_{p} \rightarrow \mathbf{S} \Sigma_{p}
$$

of the considered boundary operator to be an isomorphism from the subspace of initial conditions of those solutions, i.e.,

$$
\left\{\eta \in \mathbf{S} \Sigma_{p}: i \gamma(N) \gamma(u) \eta=-|u| \eta\right\} \subset \mathbf{S} \Sigma_{p}
$$

onto the subspace range $\left(P_{ \pm}\right)$, for each $p \in \Sigma$ and each nontrivial $u \in T_{p} \Sigma$. Since these two subspaces have the same dimension,

$$
\frac{1}{2} \operatorname{dim} \mathbf{S} \Sigma_{p}
$$

it is sufficient to prove that this linear map is injective. But, if $\eta \in \mathbf{S} \Sigma_{p}$ is one of these initial conditions and $P_{ \pm} \eta=0$, we have that $\gamma(u) \eta=\mp|u| \eta$. As $\gamma(u)^{2}=-|u|^{2} \mathrm{Id}$, this implies $\eta=0$, hence the operators $P_{ \pm}$provide elliptic boundary conditions for the Dirac operator $\bar{D}$ of $\Omega$. 
REMARK 1 . The classical local boundary condition ensures that the Dirac operator $\bar{D}$ is selfadjoint, but it doesn't exist in all dimensions and there are topological obstructions for its existence when the dimension of $\Omega$ is odd [BW, GLP, HMZ2, Se]. Our new local boundary condition exists in all dimension without any obstruction. But the following formula

$$
\int_{\Omega}\langle\bar{D} \psi, \varphi\rangle d \Omega-\int_{\Omega}\langle\psi, \bar{D} \varphi\rangle d \Omega=\int_{\Sigma}\langle\psi, \gamma(N) \varphi\rangle d \Sigma
$$

shows that the conditions provided by $P_{ \pm}$do not imply that $\bar{D}$ is selfadjoint.

Corollary 4. The following inhomogeneous problem for the Dirac operator $\bar{D}$ of a compact domain $\Omega$ in a Riemannian spin manifold $M$, with boundary a hypersurface $\Sigma$

$$
\begin{cases}\bar{D} \psi=\Psi & \text { on } \Omega \\ P_{ \pm} \psi=0 & \text { on } \Sigma\end{cases}
$$

has a unique smooth solution for any $\Psi \in \Gamma(\mathbb{S} \Omega)$.

Proof. The two realizations of $\bar{D}$ associated with the two boundary conditions $P_{ \pm}$are the two bounded operators

$$
\bar{D}_{ \pm}: \operatorname{dom} \bar{D}_{ \pm}=\left\{\psi \in H^{1}(\mathbb{S} \Omega): P_{ \pm} \psi_{\mid \Sigma}=0\right\} \longrightarrow L^{2}(\mathbb{S} \Omega)
$$

where $H^{1}$ stands for the Sobolev space of $L^{2}$-spinors with weak $L^{2}$ covariant derivatives (recall that such spinors have a well defined $L^{2}$ trace on $\Sigma$ ). From (5.2), it follows that $\bar{D}_{ \pm}^{*}=\bar{D}_{\mp}$. Moreover, if $\psi \in \operatorname{dom} \bar{D}_{ \pm}$belongs to $\operatorname{ker} \bar{D}_{ \pm}$, by taking $\varphi=i \psi$ in (5.2) (note that this formula is valid even for weak spinor fields), and recalling that the metric on $\mathbb{S} \Omega$ is Hermitian, we have

$$
0=2 \int_{\Omega}\langle\bar{D} \psi, i \psi\rangle d \Omega=\int_{\Sigma}\langle\psi, i \gamma(N) \psi\rangle d \Sigma=\mp \int_{\Sigma}|\psi|^{2} d \Sigma .
$$

Then one gets a weak harmonic spinor by extending $\psi$ by zero to a compact manifold containing $\Omega$ and so, regularity for the Dirac operator on compact manifolds and the unique continuation property (cf. Theorem 8.2 in [BW]) say that $\psi$ vanishes on all of $\Omega$. Then

$$
\operatorname{ker} \bar{D}_{ \pm}=\{0\} \quad \text { and } \quad \operatorname{coker} \bar{D}_{ \pm} \cong \operatorname{ker} \bar{D}_{\mp}=\{0\}
$$

Then the two realizations $\bar{D}_{ \pm}$are invertible operators, hence if $\Psi \in \Gamma(\mathbb{S} \Omega)$ is a smooth spinor field on $\Omega$, there exists a unique solution $\psi \in H^{1}(\mathbb{S} \Omega)$ of (5.3). Now, the ellipticity proved in Proposition 3 implies (cf. Chapter 19 in [BW]) the required smoothness for the solution $\psi$.

REMARK 2. When the dimension $n+1$ of the manifold $M$ is odd, Proposition 2 and Corollary 4 give the existence, uniqueness and regularity to the problem

$$
\left\{\begin{array}{l}
\bar{D} \psi=\Psi \\
\psi_{\mid \Sigma} \in \mathbf{S} \Sigma^{\mp}
\end{array} \text { on } \Omega\right.
$$

When $n+1$ is even, if we decompose the spinor fields $\psi$ and $\Psi$ according to the decomposition (2.2) of $\Omega$, Corollary 4 solves the following boundary first order system

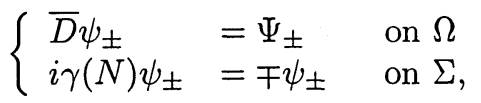


where now all the involved fields have a fixed chirality.

Corollary 5. Let $\Omega$ a compact Riemannian spin manifold with boundary a hypersurface $\Sigma$. If $\varphi \in \Gamma(\mathbf{S} \Sigma)$ is a smooth spinor field in the induced Dirac bundle and $\Psi \in \Gamma(\mathbb{S} \Omega)$, then the system

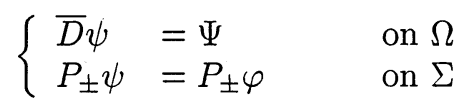

has a unique smooth solution $\psi \in \Gamma(\mathbb{S} \Omega)$.

Proof. Extend $\varphi$ to a spinor field $\widehat{\varphi} \in \Gamma(\mathbb{S} \Omega)$ and put $\widehat{\psi}=\psi-\widehat{\varphi}$. Then solve

$$
\begin{cases}\bar{D} \widehat{\psi}=-\bar{D} \widehat{\varphi} & \text { on } \Omega \\ P_{ \pm} \widehat{\psi}=0 & \text { on } \Sigma\end{cases}
$$

using Corollary 4.

6. Extrinsic conformal lower bounds for the Dirac operator. Now, we will use the Reilly type inequality (4.9) for a compact Riemannian spin manifold with boundary $\Omega$ in order to deduce lower bounds for the eigenvalues of the classical Dirac operator $D$ of the boundary hypersurface $\partial \Omega=\Sigma$. We will start by taking an eigenspinor field $\varphi \in \Gamma(\mathbf{S} \Sigma)$ for the Dirac operator $\mathbf{D}$ of the induced bundle on the hypersurface corresponding to the first eigenvalue $\lambda_{1} \geq 0$. (Recall that, from Proposition 2, $\lambda_{1}$ is also the smallest nonnegative eigenvalue of the intrinsic Dirac operator $D$ of the hypersurface.) Let $f$ be any positive smooth function defined on $\Omega$. We will consider the conformal metric $\langle,\rangle^{\star}$ defined in (4.5) and pose the following boundary problem

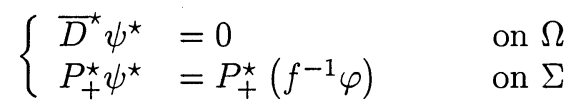

for the conformally modified Dirac operator $\bar{D}^{\star}$ and the associated boundary condition $P_{+}^{\star}$. Corollary 5 asserts that this problem has a unique smooth solution $\psi^{\star} \in \Gamma(\mathbb{S} \Omega)$. Recall that inequality (4.9) is valid for spinor fields $\psi$ and $\psi^{\star}$ on $\Omega$ such that $\psi=f \psi^{\star}$ (see 4.8) for any positive function on $\Omega$. Putting the smooth solution of the boundary value problem (6.1) in inequality (4.9), yields to the following key inequality

$$
\int_{\Sigma}\left(\langle\mathbf{D} \psi, \psi\rangle-\frac{n}{2}|\psi|^{2} f^{-1} B f\right) d \Sigma \geq \frac{1}{4} \int_{\Omega}|\psi|^{2} f^{-1} L f d \Omega
$$

The main result of this paper will rely on inequality (6.2). For this, we need some elementary lemmas.

LEMMA 6. For every smooth field $\psi \in \Gamma(\mathbf{S} \Sigma)$ we have

$$
\int_{\Sigma}\langle\mathbf{D} \psi, \psi\rangle d \Sigma=2 \Re \int_{\Sigma}\left\langle\mathbf{D} P_{+} \psi, P_{-} \psi\right\rangle d \Sigma
$$

Proof. We have the orthogonal decomposition $\psi=P_{+} \psi+P_{-} \psi$. Moreover, from definition (5.1) and Lemma 1, one immediately shows that

$$
\mathbf{D} P_{ \pm}=P_{\mp} \mathbf{D}
$$


Hence

$$
\langle\mathbf{D} \psi, \psi\rangle=\left\langle\mathbf{D} P_{+} \psi, P_{-} \psi\right\rangle+\left\langle\mathbf{D} P_{-} \psi, P_{+} \psi\right\rangle \text {. }
$$

We conclude by noting that $\Sigma$ is compact and the operator $\mathbf{D}$ is formally selfadjoint.

Lemma 7. Let $\varphi \in \Gamma(\mathbf{S} \Sigma)$ be an eigenspinor field for $\mathbf{D}$ associated with a nonzero eigenvalue. Then

$$
\int_{\Sigma}\left|P_{+} \varphi\right|^{2} d \Sigma=\int_{\Sigma}\left|P_{-} \varphi\right|^{2} d \Sigma
$$

Proof. From $\mathbf{D} \varphi=\lambda \varphi$ for a certain $\lambda \in \mathbb{R}^{*}$ and the anticommutativity relation (6.3) we obtain $\mathbf{D} P_{ \pm} \varphi=\lambda P_{\mp} \varphi$, and so $\left\langle\mathbf{D} P_{ \pm} \varphi, P_{\mp} \varphi\right\rangle=\lambda\left|P_{\mp} \varphi\right|^{2}$. Now it suffices to integrate and use again that $\mathbf{D}$ is formally selfadjoint.

LEMma 8. Let $\varphi \in \Gamma(\mathbf{S} \Sigma)$ be an eigenspinor field for $\mathbf{D}$ associated with the first nonzero eigenvalue $\lambda_{1} \in \mathbb{R}^{*}$ and $\psi^{\star}$ the unique solution of the boundary value problem (6.1). Set $\psi=f \psi^{\star}$ as in (4.8). Then

$$
\lambda_{1} \int_{\Sigma}|\psi|^{2} d \Sigma-\frac{n}{2} \int_{\Sigma}|\psi|^{2} f^{-1} B f d \Sigma \geq \frac{1}{4} \int_{\Omega}|\psi|^{2} f^{-1} L f d \Omega
$$

Moreover, equality holds if and only if the spinor $\psi^{\star}$ is a (harmonic) twistor-spinor, i.e., parallel, with respect to the modified metric and either $\lambda_{1}=0$ or $\psi=\varphi$ along the boundary hypersurface $\Sigma$.

Proof. By (6.1), we have $P_{+}^{\star} \psi^{\star}=P_{+}^{\star}\left(f^{-1} \varphi\right)$. By conformal invariance of the zero order operators $P_{ \pm}^{\star}$, it follows $P_{+}\left(f^{-1} \psi\right)=P_{+}\left(f^{-1} \varphi\right)$, and so

$$
P_{+} \psi=P_{+} \varphi \text {. }
$$

Using this fact with Lemma 6, we get

$$
\int_{\Sigma}\langle\mathbf{D} \psi, \psi\rangle d \Sigma=2 \Re \int_{\Sigma}\left\langle\mathbf{D} P_{+} \varphi, P_{-} \psi\right\rangle d \Sigma
$$

As in Lemma 7, we have $\mathrm{D} P_{+} \varphi=\lambda_{1} P_{-} \varphi$, hence

$$
\int_{\Sigma}\langle\mathbf{D} \psi, \psi\rangle d \Sigma=2 \Re \lambda_{1} \int_{\Sigma}\left\langle P_{-} \varphi, P_{-} \psi\right\rangle d \Sigma
$$

We now observe that, since $\lambda_{1} \geq 0$, one has

$$
2 \lambda_{1} \Re\left\langle P_{-} \varphi, P_{-} \psi\right\rangle \leq \lambda_{1}\left(\left|P_{-} \varphi\right|^{2}+\left|P_{-} \psi\right|^{2}\right)
$$

and equality occurs if and only if either $\lambda_{1}=0$ or $P_{-} \varphi=P_{-} \psi$. By integrating (6.6) and using Lemma 7 , it follows that

$$
2 \lambda_{1} \Re \int_{\Sigma}\left\langle P_{-} \varphi, P_{-} \psi\right\rangle d \Sigma \leq \lambda_{1}\left(\int_{\Sigma}\left|P_{+} \varphi\right|^{2} d \Sigma+\int_{\Sigma}\left|P_{-} \psi\right|^{2} d \Sigma\right)
$$

which together with the boundary condition (6.5), implies

$$
\int_{\Sigma}\langle\mathbf{D} \psi, \psi\rangle d \Sigma \leq \lambda_{1} \int_{\Sigma}|\psi|^{2} d \Sigma
$$


Finally, the combination of inequalities (6.7) and (6.2) yield to (6.4). The last statement is straightforward.

Now a suitable choice of the function $f$ will lead to one of the main results of this paper, which could be thought of as an analogue for embedded hypersurfaces of the so called Hijazi inequality [Hij1].

THEOREM 9. Let $\Sigma$ be a compact hypersurface of dimension $n \geq 2$ of a Riemannian spin manifold $M$ bounding a compact domain $\Omega$. The lowest nonnegative eigenvalue $\lambda_{1}$ of the Dirac operator on $\Sigma$ satisfies

$$
\lambda_{1} \geq \frac{n}{2} \nu_{1}(B)
$$

where $\nu_{1}(B)$ is the first eigenvalue of the boundary linear operator $B$ acting on functions $f$ defined on $\Omega$ with $L f=0$, where $L$ is the conformal Laplacian of $M$ (see (4.6)). Moreover, if equality holds, then $\Omega$ is conformally equivalent to a Riemannian spin manifold with nontrivial parallel spinors (hence Ricci flat) and the eigenspace corresponding to $\lambda_{1}$ is isomorphic to the space of restrictions to $\Sigma$ of parallel spinors.

Proof. First, recall that the eigenvalue problem

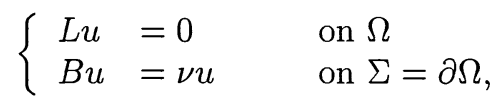

appearing in the statement of this theorem, was introduced by Escobar in [Es1] in the context of the Yamabe problem for manifolds with boundary and that $L$ and $B$ are the operators defined in (4.6). The corresponding first eigenvalue $\nu_{1}(B)$, whose variational characterization is given by

$$
\nu_{1}(B)=\inf _{f \in C^{1}(\bar{\Omega}), f \neq 0} \frac{\int_{\Omega}\left(\frac{2}{n-1}|\bar{\nabla} f|^{2}+\frac{1}{2 n} \bar{R} f^{2}\right) d \Omega+\int_{\Sigma} H f^{2} d \Sigma}{\int_{\Sigma} f^{2} d \Sigma},
$$

is not necessarily finite (see Addendum to [Es1]), although reasonable geometric assumptions on $\Omega$ (for example, nonnegative scalar curvature), immediately imply its finiteness. Obviously, if $\nu_{1}(B)=-\infty$, the theorem is true. Hence we will suppose that $\nu_{1}(B)$ is a finite real number.

In this case, Escobar proved that the sign of $\nu_{1}$ is invariant under conformal change of the metric on $\Omega$ and an associated eigenfunction $f$ has to be positive (Proposition 1.3 in [Es1]). Moreover, Escobar proved that $\nu_{1}$ is positive (resp. zero or negative) if and only if there exists a conformally related metric on $\Omega$ with zero scalar curvature and such that the boundary $\Sigma$ has positive (resp. identically zero or negative) mean curvature, or equivalently, there is a conformal metric on $\Omega$ with positive (resp. identically zero or negative) scalar curvature and minimal boundary. In many cases, the mean curvature turns out to be constant.

Now we choose the function $f$ in (6.4) to be a positive eigenfunction associated with $\nu_{1}(B)$. Hence,

$$
\left(\lambda_{1}-\frac{n}{2} \nu_{1}(B)\right) \int_{\Sigma}|\psi|^{2} d \Sigma \geq 0
$$

which is precisely the desired inequality (note that $\psi$ cannot vanish identically on $\Sigma$ since $P_{+} \psi$ is an eigenspinor field).

It only remains to examine the equality case. If $\lambda_{1}=(n / 2) \nu_{1}(B)$, then equality occurs in (6.4) and so the nontrivial spinor field $\psi^{\star}$ is parallel w.r.t $\langle,\rangle^{\star}$ and either 
$\lambda_{1}=\nu_{1}(B)=0$ or $\psi_{\mid \Sigma}=\varphi$. But, if the first possibility holds, by (6.3) we conclude that $\mathbf{D} P_{ \pm} \varphi=0$. Then, if we repeat the same argument for $P_{ \pm} \varphi$ instead of $\varphi$ (when we have the - sign, we must address the corresponding boundary problem with boundary condition $P_{-}^{\star}$ ), we also obtain $\psi_{\mid \Sigma}=\varphi$.

Conversely, let $\psi^{\star}$ be a nontrivial parallel spinor with respect to the metric $\langle,\rangle^{\star}$. Applying (3.7) to this conformal metric, we get

$$
\mathbf{D}^{\star} \psi^{\star}=\frac{n}{2} H^{\star} \psi^{\star}
$$

Now we define $\psi$ by the relation $\psi=f \psi^{\star}$ and take into account (4.3), (4.4) and (4.6). Then,

$$
\mathbf{D} \psi=\frac{n}{2}\left(f^{-1} B f\right) \psi
$$

which by the choice of $f$, could be written as

$$
\mathbf{D} \psi=\frac{n}{2} \nu_{1}(B) \psi=\lambda_{1} \psi
$$

REMARK 3. Note that if equality is achieved in (9), there exists a nontrivial parallel spinor on $\left(\Omega,\langle,\rangle^{\star}\right)$.

COROLlARY 10. If the boundary of a compact Riemannian spin manifold admits a nontrivial harmonic spinor, then there exists a metric in the conformal class with negative or zero scalar curvature and the boundary has to be minimal.

REMARK 4. It is clear that, if $\bar{R} \geq 0$, then $\nu_{1}(B) \geq \inf _{\Sigma} H$. Equality occurs if and only if the conformal factor $f$ is constant, $\bar{R} \equiv 0$, and $H$ is constant. As a consequence, we get Theorem 6 in [HMZ1]. On the other hand, there are examples where only (6.8) is significant. In fact, if $\Sigma$ is a 2-dimensional torus embedded in $\mathbb{R}^{3}$, the Gauss-Bonnet theorem implies that $\inf _{\Sigma} R<0$. But, in the case of a torus of revolution in $\mathbb{R}^{3}$, obtained by rotating a circle of radius $r$ whose center is at distance $a>r$ of the axis of rotation, we have that $H>0$ if (and only if) $a>2 r$. These tori of revolution provide examples for which Theorem 6 in [HMZ1] gives no information, while inequality (6.8) is still significant. If $a=\sqrt{2} r$ the corresponding torus of revolution is the stereographic projection of a minimal torus in the threesphere (the Clifford torus). Hence, for the Clifford torus $\nu_{1}(B)>0$ while $\inf _{\Sigma} H<0$.

It was also Escobar (see, for example [Es1] and references therein) who introduced the following Sobolev quotient, called the boundary Yamabe conformal invariant

$$
\mathcal{Q}(\Omega, \Sigma)=\inf _{f \in C^{1}(\bar{\Omega}), f \neq 0} \frac{\int_{\Omega}\left(\frac{2}{n-1}|\bar{\nabla} f|^{2}+\frac{1}{2 n} \bar{R} f^{2}\right) d \Omega+\int_{\Sigma} H f^{2} d \Sigma}{\left(\int_{\Sigma} f^{\frac{2 n}{n-1}} d \Sigma\right)^{\frac{n-1}{n}}} .
$$

He proved that $\mathcal{Q}(\Omega, \Sigma)$ has the same sign as $\nu_{1}(B)$ and it is invariant with respect to conformal changes of the metric on $\Omega$. The Hölder inequality applied to an eigenfunction $f$ associated with $\nu_{1}$ gives

$$
\nu_{1}(B) \geq \frac{\mathcal{Q}(\Omega, \Sigma)}{\operatorname{vol}(\Sigma)^{\frac{1}{n}}}
$$


and equality implies that $f$ is constant. Thus, from Theorem 9 one has a conformal lower bound for the product $\lambda_{1} \operatorname{vol}(\Sigma)^{1 / n}$.

COROLlaRY 11. Let $\Sigma$ be a compact hypersurface of dimension $n \geq 2$ of a Riemannian spin manifold $M$ bounding a compact domain $\Omega$. Then the lowest nonnegative eigenvalue $\lambda_{1}$ of the Dirac operator on $\Sigma$ satisfies

$$
\lambda_{1} \operatorname{vol}(\Sigma)^{\frac{1}{n}} \geq \frac{n}{2} \mathcal{Q}(\Omega, \Sigma)
$$

where $\mathcal{Q}(\Omega, \Sigma)$ is the boundary Yamabe conformal invariant. Moreover, if equality holds, then the eigenspace associated with $\lambda_{1}$ consists of restrictions of parallel spinors on $\Omega$.

REMARK 5. For compact (immersed) surfaces in the Euclidean space, it is known (see $[\mathrm{An}, \mathrm{Bm}, \mathrm{B} \ddot{2} 2]$ and $[\mathrm{AF}]$ for generalizations) that

$$
\lambda_{1}^{2} \operatorname{area}(\Sigma) \leq \int_{\Sigma} H^{2} d \Sigma .
$$

Then, for embedded surfaces in $\mathbb{R}^{3}$, one has

$$
\mathcal{Q}^{2}(\Omega, \Sigma) \leq \lambda_{1}^{2} \operatorname{area}(\Sigma) \leq \int_{\Sigma} H^{2} d \Sigma,
$$

where $\Omega$ is the enclosed domain. That is, the scale free quantity $\lambda_{1}^{2} \operatorname{area}(\Sigma)$ is between two extrinsic conformal invariants: the Yamabe number and the Willmore functional.

\section{REFERENCES}

[AF] I. Agricola, T. FRIEDRICH, Upper bounds for the first eigenvalue of the Dirac operator on surfaces, Journ. Geom. Phys., 301 (1999), pp. 1-22.

[Al] A.D. Alexandrov, A characteristic property of spheres, Ann. Mat. Pura Appl., 58 (1962), pp. 303-315.

[An] N. ANGHEL, Extrinsic upper bounds for eigenvalues of Dirac-type operators, Proc. AMS, 117 (1993), pp. 501-509.

[Ar] H. ARAUJo, Critical points of the total scalar curvature plus total mean curvature, thesis, Cornell University, May 2001.

[Bä1] C. BÄr, Metrics with Harmonic Spinors, Geometric And Functional Analysis, 6 (1996), pp. 899-942.

[Bä2] C. BÄR, Extrinsic bounds of the Dirac operator, Ann. Glob. Anal. Geom., 16 (1998), pp. $573-596$.

[Bm] H. BAUM, An upper bound for the first eigenvalue of the Dirac operator on compact spin manifolds, Math. Z., 206 (1991), pp. 409-422.

[BFGK] H. Baum, T. Friedrich, R. Grünewald, I. Kath, Twistor and Killing Spinors on Riemannian Manifolds, Seminarbericht, Humboldt-Universität zu Berlin, 108 (1990).

[BW] B. Booss-Bavnbek, K.P. Wojciechowski, Elliptic Boundary Problems for the Dirac Operator, Birkhäuser, Basel, 1993.

[BHMM] J.P. Bourguignon, O. Hijazi, J.-L. Milhorat, A. Moroianu, A Spinorial Approach to Riemannian and Conformal Geometry, Monograph (In Preparation).

[Bu] J. Bureš, Dirac operators on hypersurfaces, Comment. Math. Univ. Carolin., 34 (1993), No. 2, pp. 313-322.

[Es1] J.F. EscoBAR, Conformal deformation of a Riemannian metric to a scalar flat metric with constant mean curvature on the boundary, Ann. of Math., 136 (1992), pp. 1-50. (Addendum in 139 (1994), pp. 749-750.)

[Es2] J.F. EscoBAR, The geometry of the first non-zero Stekloff eigenvalue, J. Funct. Anal, 150 (1997), No. 2, pp. 544-556.

[Es3] J.F. Escobar, An isoperimetric inequality and the first Steklov eigenvalue, J. Funct. Anal, 165 (1999), No. 1, pp. 101-116. 
[Es4] J.F. Escobar, A comparison theorem for the first non-zero Steklov eigenvalue, J. Funct. Anal, 178 (2000), No. 1, pp. 143-155.

[Fr] T. FRIEDRICH, Der erste Eigenwert des Dirac-Operators einer kompakten Riemannschen Mannifaltigkeit nicht negativer Skalarkrümmung, Math. Nach., 97 (1980), pp. 117146.

[GLP] P.B. Gilkey, J.V. Leahy, J. PARK, Spectral Geometry, Riemannian Submersions and the Gromov-Lawson Conjecture, Studies in Advanced Mathematics, Chapman \& Hall/Crc, Boca Raton, 1999.

[Hij1] O. HiJAzI, A conformal lower bound for the smallest eigenvalue of the Dirac operator and Killing spinors, Commun. Math. Phys., 104 (1986), pp. 151-162.

[Hij2] O. HijazI, Première valeur propre de l'opérateur de Dirac et nombre de Yamabe, C. R. Acad. Sci. Paris, 313 (1991), pp. 865-868.

[HMZ1] O. HiJazI, S. Montiel, X. Zhang, Dirac operator on embedded hypersurfaces, Math. Res. Lett., 8 (2001), pp. 195-208.

[HMZ2] O. HiJazi, S. Montiel, X. Zhang, Eigenvalues of the Dirac Operator on Manifolds with Boundary, Commun. Math. Phys., 221 (2001), pp. 255-265.

[Hit] N. Hitchin, Harmonic spinors, Adv. in Math., 14 (1974), pp. 1-55.

[Hö] L. Hörmander, The Analysis of Linear Partial Differential Operators III, Springer, Berlin, 1985.

[LM] H.B. Lawson, M.L. Michelsohn, Spin Geometry, Princeton Math. Series, vol. 38, Princeton University Press, 1989.

[Li] A. Lichnerowicz, Spineurs harmoniques, C.R. Acad. Sci. Paris, 257 (1963), Série I, pp. 7-9.

[Lo] J. Lot, Eigenvalue Bounds for the Dirac operator, Pacific J. Math., 125 (1986), pp. $117-126$.

[Se] R. SEeley, Singular integrals and boundary problems, Amer. J. Math., 88 (1966), pp. 781-809.

[Tr] A. Trautman, The Dirac Operator on Hypersurfaces, Acta Phys. Plon., B 26 (1995), pp. 1283-1310. 